\title{
Cortical Slow Oscillatory Activity Is Reflected in the Membrane Potential and Spike Trains of Striatal Neurons in Rats with Chronic Nigrostriatal Lesions
}

\author{
Kuei Y. Tseng, Fernando Kasanetz, Lucila Kargieman, Luis A. Riquelme, and M. Gustavo Murer \\ Departamento de Fisiología, Facultad de Medicina, Universidad de Buenos Aires, Paraguay 2155, Buenos Aires 1121, \\ Argentina
}

Neurons in the basal ganglia output nuclei display rhythmic burst firing after chronic nigrostriatal lesions. The thalamocortical network is a strong endogenous generator of oscillatory activity, and the striatum receives a massive projection from the cerebral cortex. Actually, the membrane potential of striatal projection neurons displays periodic shifts between a very negative resting potential (down state) and depolarizing plateaus (up states) during which they can fire action potentials. We hypothesized that an increased excitability of striatal neurons may allow transmission of cortical slow rhythms through the striatum to the remaining basal ganglia in experimental parkinsonism. In vivo intracellular recordings revealed that striatal projection neurons from rats with chronic nigrostriatal lesions had a more depolarized membrane potential during both the down and up states and an increased firing probability during the up events. Furthermore, lesioned rats had signifi-

It is conventionally accepted that a substantial part of the influence of dopamine (DA) on sensorimotor and cognitive functions is related to its modulatory effects on striatal processing of cortical input (Albin et al., 1989; Hollerman et al., 2000). Dopamine inputs from the substantia nigra converge with glutamatergic inputs from the cerebral cortex at the dendrites of striatal projection neurons (Smith et al., 1998), which are called "medium spiny neurons" (MSNs). In vivo intracellular recordings from MSNs revealed that their membrane potential alternates between two steady-state values. A very polarized resting potential ("down state") is interrupted by periods of sustained depolarization ("up states") lasting a few hundred milliseconds (Wilson and Groves, 1981; Calabresi et al., 1990; Wilson, 1993). There is evidence supporting the theory that the up events are driven by excitatory inputs from the cerebral cortex and thalamus (Wilson et al., 1983; Wilson 1993; Plenz and Aertsen, 1996). In vitro studies indicate that the ionic conductances shaping the fluctuating membrane potential of MSNs are modulated by DA (Nicola et al., 2000). The up events are currently perceived as "enabling states," during

Received March 30, 2001; revised May 17, 2001; accepted May 31, 2001.

This work was supported by the Ministerio de Salud y Acción Social de la Nación (Beca Carrillo-Oñativia), Fundación Antorchas, Consejo Nacional de Investigaciones Científicas y Técnicas, y Universidad de Buenos Aires (Argentina). We thank Dr. Patricio O'Donnell for helpful comments on an earlier version of this manuscript, Dr. José L. Ferrán for excellent assistance with microphotography and artwork, and Departamento de Ciencias Biológicas, Facultad de Ciencias Exactas y Naturales, Universidad de Buenos Aires, for their help with time series analysis.

Correspondence should be addressed to Kuei Y. Tseng, Departamento de Fisiología, Facultad de Medicina, Universidad de Buenos Aires, Paraguay 2155, Buenos Aires (1121), Argentina. E-mail: neurofis@fmed.uba.ar.

Copyright (C) 2001 Society for Neuroscience 0270-6474/01/216430-10\$15.00/0 cantly fewer silent neurons than control rats. Simultaneous recordings of the frontal electrocorticogram and membrane potential of striatal projection neurons revealed that the signals were oscillating synchronously in the frequency range $0.4-2 \mathrm{~Hz}$, both in control rats and rats with chronic nigrostriatal lesions. Spreading of the slow cortical rhythm is limited by the very low firing probability of control rat neurons, but a slow oscillation is well reflected in spike trains of $\sim 60 \%$ of lesioned rat neurons. These findings provide in vivo evidence for a role of dopamine in controlling the flow of cortical activity through the striatum and may be of outstanding relevance for understanding the pathophysiology of Parkinson's disease.

Key words: striatum; cerebral cortex; dopamine; in vivo intracellular recording; Parkinson's disease; neuronal firing patterns which synchronously depolarized MSNs translate afferent activity into sequences of action potentials, allowing transmission of information to structures receiving striatal projections (Wilson, 1993; O’Donnell and Grace, 1995).

Extracellular single unit recordings established that after nigrostriatal system lesions, the firing pattern of neurons in nuclei receiving strong striatal innervation, like the globus pallidus (GP) and substantia nigra pars reticulata (SNpr), shifts from a regular pattern to a rhythmic burst firing mode (Pan and Walters, 1988; MacLeod et al., 1990; Murer et al., 1997; Ni et al., 2000). Recent studies from our laboratory indicate that as much as $40 \%$ of SNpr units recorded from 6-hydroxydopamine (6-OHDA)-lesioned rats display rhythmic $\sim 1 \mathrm{~Hz}$ firing rate modulations (Tseng et al., 2001). Our findings showing a strong modulatory effect of intrastriatally administered DA receptor agonists on the firing pattern of SNpr units in 6-OHDA-lesioned rats support a role of the striatum in the genesis of this phenomenon (Tseng et al., 2000). Interestingly, it has been reported that the membrane potential fluctuations of MSNs display a weak periodicity, also with a frequency of $\sim 1 \mathrm{~Hz}$ (Stern et al., 1997). In slow wave sleep and anesthesia, rhythmic modulations of firing rate occur in neurons throughout the cerebral cortex and thalamus. Rhythmic activity of the thalamocortical network is reflected in the electroencephalogram (EEG) of animals and humans as oscillations of different frequencies (including a slow $\sim 1 \mathrm{~Hz}$ oscillation) that are synchronized over widespread areas of the cerebral cortex (Steriade, 1999). Therefore, we hypothesized that an altered functional state of striatal projection neurons may facilitate the transmission of slow cortical rhythms to the basal ganglia output nuclei in 
experimental parkinsonism. In this manuscript, we report the existence of a strong correlation between cortical slow rhythmic activity and membrane potential fluctuations of striatal neurons. Furthermore, we describe modifications in the membrane potential of striatal neurons from 6-OHDA-lesioned rats that may allow transmission of slow cortical rhythms toward basal ganglia output nuclei in experimental parkinsonism.

\section{MATERIALS AND METHODS}

Male adult Sprague Dawley rats, weighing 190-220 gm, were randomly assigned to receive a 6-OHDA lesion or a sham lesion. The animals were maintained on a $12 \mathrm{hr}$ light/dark cycle with food and tap water available ad libitum until the time of the experiment. In vivo intracellular recordings of striatal neurons were performed 6-10 weeks after the lesion.

Lesions. A severe unilateral lesion of mesencephalic dopaminergic neurons was obtained with the neurotoxin 6-OHDA hydrobromide (Sigma, St. Louis, MO), as reported previously (Murer et al., 1997). Briefly, the rats were anesthetized with pentobarbital $(50 \mathrm{mg} / \mathrm{kg}$, i.p.), and with the aid of a stereotaxic instrument (David Kopf Instruments, Tujunga, $\mathrm{CA})$, they were injected with the neurotoxin ( $8 \mu \mathrm{g}$ free base in $4 \mu \mathrm{l}$ of $0.1 \%$ ascorbic acid) at the medial forebrain bundle (stereotaxic coordinates: anterior (A), -4.3 from bregma; lateral (L), 1.6; $\mathrm{H}, 8.3$ below the cortical surface) (Paxinos and Watson, 1997). The control group received only the ascorbic acid solution. The effectiveness of the lesion was evaluated with the stepping test (Olsson et al., 1995; Chang et al., 1999) and further confirmed postmortem by means of immunohistochemistry (Murer et al., 1997). The stepping test was repeated three times between days 15 and 20 after surgery in all the rats. Only those 6-OHDA-lesioned rats that showed less than two adjusting steps with the forelimb contralateral to the lesion during each trial were selected for the experiments (see Fig. 1).

Electrophysiological recordings. The rats were anesthetized with urethane $(1.2 \mathrm{gm} / \mathrm{kg}$, i.p. $)$, treated with a local anesthetic on the scalp and pressure points, and secured to the stereotaxic frame. Temperature was maintained between 36 and $37^{\circ} \mathrm{C}$ with a heating pad. Additional urethane was administered throughout the experiment as necessary to maintain a constant level of anesthesia, as determined by testing the hindlimb compression reflex, and electrocorticographic recordings when available (customarily, supplements of $0.4 \mathrm{gm} / \mathrm{kg}$, s.c., every 3-4 hr).

A concentric bipolar stimulating electrode (SNE-100; Better Hospital Equipment, Rockville Centre, NY) was placed in the frontal cortex (A, $3 \mathrm{~mm}$ anterior to the bregma; L, $1.5 \mathrm{~mm}$; and $\mathrm{H}, 2.5 \mathrm{~mm}$ below the cortical surface) (Paxinos and Watson, 1997) to allow delivery of 300 $\mu$ sec square wave pulses, $300-500 \mu \mathrm{A}$ in amplitude $(10-15$ pulses at 0.5 $\mathrm{Hz}$ ). In many experiments, an additional concentric bipolar electrode was located $1 \mathrm{~mm}$ lateral to the stimulating electrode, with the tip inserted $\sim 1.5 \mathrm{~mm}$ below the cortical surface, to obtain a differential recording of the electrocorticogram (ECoG). The cortical signal was filtered (0.1-300 $\mathrm{Hz}$ ), amplified (ER-98; NeuroData, Delaware Water Gap, PA), and sent to an A/D converter (DigiData 1200; Axon Instruments, Foster City, CA).

Intracellular recordings were obtained from a striatal region located $1.0 \mathrm{~mm}$ anterior to bregma, $2.5-3.0 \mathrm{~mm}$ lateral to the midline, and 3.0-5.0 mm below the cortical surface (Paxinos and Watson, 1997). The microelectrodes were pulled (Rhema-Labortechnik microelectrode puller; Campden Instruments, Loughborough, UK) from $1.2 \mathrm{~mm}$ outer diameter borosilicate glass capillaries (WPI, Sarasota, FL) and filled with 2 M K-acetate and 2\% Neurobiotin (Research Biochemicals, Natick, MA); they had resistances ranging from 60 to $130 \mathrm{M} \Omega$. The recorded signal was sent to a conventional bridge amplifier (IR-283; NeuroData) and to the A/D converter. The microelectrode was advanced with a hydraulic micromanipulator through the cortex up to the striatum (2.5-3 $\mathrm{mm}$ below the cortical surface), while continuously measuring electrode resistance by passing $0.5 \mathrm{nA}, 100 \mathrm{msec}$ current pulses. The microelectrode was left in place for 20-30 min and then slowly advanced through the striatum until a neuron was impaled. Recordings had to fulfill all of the following criteria to be included in the study: (1) resting membrane potential of at least $-55 \mathrm{mV}$; (2) action potential amplitude $>45 \mathrm{mV}$ measured from threshold and duration inferior to $1 \mathrm{msec}$ measured at half maximal amplitude; and (3) input resistance $>20 \mathrm{M} \Omega$. All of these parameters had to remain stable for at least $10 \mathrm{~min}$ (without any hyperpolarizing current). Input resistance was estimated from the membrane potential measured $80-90 \mathrm{msec}$ after the onset of small amplitude hyperpolarizing or depolarizing (subthreshold) current pulses. Ordi- narily, several epochs of spontaneous activity lasting 1-2 min could be recorded, and the activity evoked by cortical stimulation could be evaluated at least twice at intervals of 5-10 min. All signals were acquired with Axoscope 1.1 (Axon Instruments) at a sampling rate of $10 \mathrm{kHz}$.

After completion of the experimental manipulations, we attempted to label the neurons with Neurobiotin by passing $1 \mathrm{nA}, 300 \mathrm{msec}$ positive current pulses at a frequency of $2 \mathrm{~Hz}$ for at least $20 \mathrm{~min}$ (Kita and Armstrong, 1991). The rats were transcardially perfused with cold saline, followed by $4 \%$ paraformaldehyde in PBS. The brain was removed, stored overnight in the same fixative, and then incubated in PBS containing $15 \%$ sucrose for $24 \mathrm{hr}$.

Tissue processing. Serial $40-\mu \mathrm{m}$-thick coronal sections were obtained from the entire forebrain and mesencephalon. The localization of the extracellular recording sites was determined from Nissl-stained sections. Neurobiotin was revealed after a slightly modified version of the protocol of Kita and Armstrong (1991). Briefly, the sections were incubated for 4 $\mathrm{hr}$ in $0.1 \mathrm{M}$ PBS containing an avidin-biotin-peroxidase complex $(1 / 100$, Vectastain Elite ABC kit; Vector Laboratories, Burlingame, CA) and $0.3 \%$ Triton $\mathrm{X}-100$, washed in $0.1 \mathrm{M}$ PBS, and incubated for $5-10 \mathrm{~min}$ in $0.05 \mathrm{M}$ sodium acetate containing $0.5 \mathrm{mg} / \mathrm{ml}$ 3, $3^{\prime}$-diaminobenzidine (Sig$\mathrm{ma}$ ), $0.8 \mathrm{mg} / \mathrm{ml}$ ammonium nickel sulfate, and $0.2 \mu \mathrm{l} / \mathrm{ml} \mathrm{H}_{2} \mathrm{O}_{2}$. Tyrosinehydroxylase $(\mathrm{TH})$ immunohistochemistry was performed on free-floating sections following a published protocol (Murer et al., 1997).

Analysis of membrane potential fluctuations and firing pattern of striatal neurons. For all the neurons included in the study, at least two $1 \mathrm{~min}$ signal segments of basal activity were analyzed. Sampling was reduced to $1000 \mathrm{~Hz}$ by using the substitute average module of Axoscope. The signal was browsed to select epochs showing a clear two-state membrane potential. These epochs were used to construct histograms displaying the amount of time spent at any given membrane potential (all-points histograms, pClamp 6; Axon Instruments). For most recorded neurons, the histogram showed a clear bimodal profile fitting to a dual-Gaussian function (see Fig. 2). Fitting was performed with the LevenbergMarquart method of nonlinear least squares (pClamp 6; Axon Instruments) (Stern et al., 1997). The voltage corresponding to the minimum reached by the function amid its peaks was used to estimate the time of state transitions in the signal. The time spent in the up and down states was estimated with custom-made software that records a state each time that voltage remains for $>100 \mathrm{msec}$ below or above the selected transitional value. This approach was preferred to the alternative of estimating time spent in the lower and upper voltage ranges of the distribution directly from the histogram, because the duration of individual states probably influences distinctively the activity of voltage-sensitive ionic conductances and firing probability (Nicola et al., 2000). The mode inside each of the two resulting series of voltage values was considered to represent the steady state reached during the down and up states.

Spectral analysis was used to characterize the periodic components in the membrane potential of striatal neurons. Spectra were computed from 1 min segments of signal (down-sampled to $1000 \mathrm{~Hz}$ ), yielding a spectral resolution of $0.017 \mathrm{~Hz}$. For the spontaneously active neurons, smoothing with a 20 points moving average was used to truncate the action potentials before performing the fast Fourier transform (FFT). Spectral densities were obtained using a Hamming window (width, 5). Relative power was calculated by normalization to the total power within the frequency range $0.017-10 \mathrm{~Hz}$ (the power of frequencies above $10 \mathrm{~Hz}$ was negligible). Peaks exceeding the $95 \%$ confidence interval of the mean relative spectral power were considered significant.

Neurons that did not spontaneously fire any action potential during the recording period were considered "silent." For the spiking neurons, the number of up states yielding at least one action potential was determined. Interspike interval (ISI) streams were obtained from the intracellular recordings by means of amplitude discrimination. Autocorrelograms were computed from 1 min segments of signal using $10 \mathrm{msec}$ bins over 1000 bins (yielding a total lag of $10 \mathrm{sec}$ ). The autocorrelograms were smoothed using a moving average method and then subjected to an FFT, yielding power spectra with $0.1 \mathrm{~Hz}$ resolution. Because the purpose of the analysis was to determine whether an $\sim 1 \mathrm{~Hz}$ rhythm can be retrieved from the spike trains of individual striatal neurons, this analysis was only performed on cells firing at rates $>1 \mathrm{~Hz}$.

Several measures were performed on the responses induced by cortical stimulation, including the time from the beginning of the stimulus artifact to the peak of the fast depolarizing postsynaptic potential (dPSP), the voltage at the peak of the fast dPSP, and the duration and amplitude of the long-lasting hyperpolarizing and depolarizing changes that follow the fast dPSP (Wilson, 1993). The same rationale that was applied to the 
analysis of naturally occurring fluctuations in membrane potential was used to examine the long-lasting changes evoked by cortical stimulation. The voltage chosen to establish the occurrence of spontaneous state transitions in the signal was used to define the beginning and end of the long-lasting changes that were evoked by cortical stimulation. The segments of signal including the long-lasting responses were cut off and used to construct histograms of the frequencies distribution of membrane potential, which showed bimodal profiles. The mode inside each part of the frequencies distribution of voltage values was considered to represent the steady state that was reached during the long-lasting hyperpolarizing and depolarizing phases of the response.

Analysis of the simultaneously acquired ECoG and striatal recordings. For each ECoG-intracellular recording pair, at least three disjoined 30 sec epochs (typically 4-6) were studied. Sampling was reduced to 1000 $\mathrm{Hz}$ as described above, and the signals were standardized. Crosscorrelograms were computed for delays of $3 \mathrm{sec}$ with a resolution of 3 msec. Peaks exceeding three correlated white noise SEs were considered significant. Cross-correlation analysis allowed estimation of the phase shift between the signals (time lag of the highest peak) and the frequency of the dominant coincident oscillatory activity (the reciprocal of the time between two successive peaks) (Lopes da Silva et al., 1989). The similarity in the oscillatory frequency content of the signals was further evaluated using spectral analysis and coherence estimation (Lopes da Silva et al., 1989). Power spectra were computed as described above (resolution for $30 \mathrm{sec}$ signal segments $=0.033 \mathrm{~Hz}$ ). Coherence was calculated from the cross-spectral density between the two signals normalized by the spectral density of each signal. Coherence attains its highest value when the phase shift and ratio between the amplitudes of the two waveforms remains constant. A significant coherence $(>0.75$ in at least three disjoined $30 \mathrm{sec}$ signal segments) (Rosenberg et al., 1989) at the dominant frequency of the cross-spectrum, was accepted as an indication of a high probability of oscillatory synchronization and allowed estimation of the phase relationship between the signals. Phase lags were calculated from portions of the phase spectrum encompassing the frequencies showing a significant coherence and a linear phase relationship between the signals (Lopes da Silva et al., 1989). Time series analysis was performed using Statistica 4.2 (Statsoft Inc., Tulsa, OK). Because the ECoG was high-pass filtered at $0.1 \mathrm{~Hz}$, the time lags were corrected using a series of coefficients that were derived from measurements of the phase lag introduced by the filter to digitally generated sinusoidal waveforms (with a $0.1 \mathrm{~Hz}$ resolution).

Statistical comparisons. The Fisher exact probability test was used to evaluate the relationship between two dichotomous variables. The Student's $t$ test was used for two-group comparisons involving a single continuous variable. If the data were not normally distributed, or had unequal variances, the Mann-Whitney $U$ test was used instead of the $t$ test. Normality was assessed with the Kolmogorov-Smirnov test, and homogeneity of variances was assessed with Levene's test. To compare sham-lesioned and 6-OHDA-lesioned rats along two or more variables, ANOVA was used. The Kruskal-Wallis ANOVA by ranks was preferred for multiple comparisons involving interrelated proportions.

\section{RESULTS}

\section{Effects of 6-OHDA-induced lesions on the membrane potential of striatal neurons}

In vivo intracellular recordings were obtained from 27 striatal neurons from 21 sham-lesioned rats and 31 striatal neurons from 24 6-OHDA-lesioned rats. All neurons successfully injected with Neurobiotin were identified as MSNs (Fig. 1). Among the seven successful Neurobiotin injections performed in control rats, all led to the labeling of a single spiny neuron. In contrast, 7 among the 11 successful Neurobiotin injections performed in 6-OHDAlesioned rats revealed two labeled spiny neurons $(p=0.013$, Fisher exact test). An increased probability of labeling more than one striatal neuron after a single intracellular Neurobiotin injection in rats with chronic nigrostriatal lesions has been reported by others (Cepeda et al., 1989; Onn and Grace, 1999) and was suggested to reflect increased "coupling" via gap junctions. Neurons recorded from control and 6-OHDA-lesioned rats did not differ in some of their basic electrophysiological parameters, like
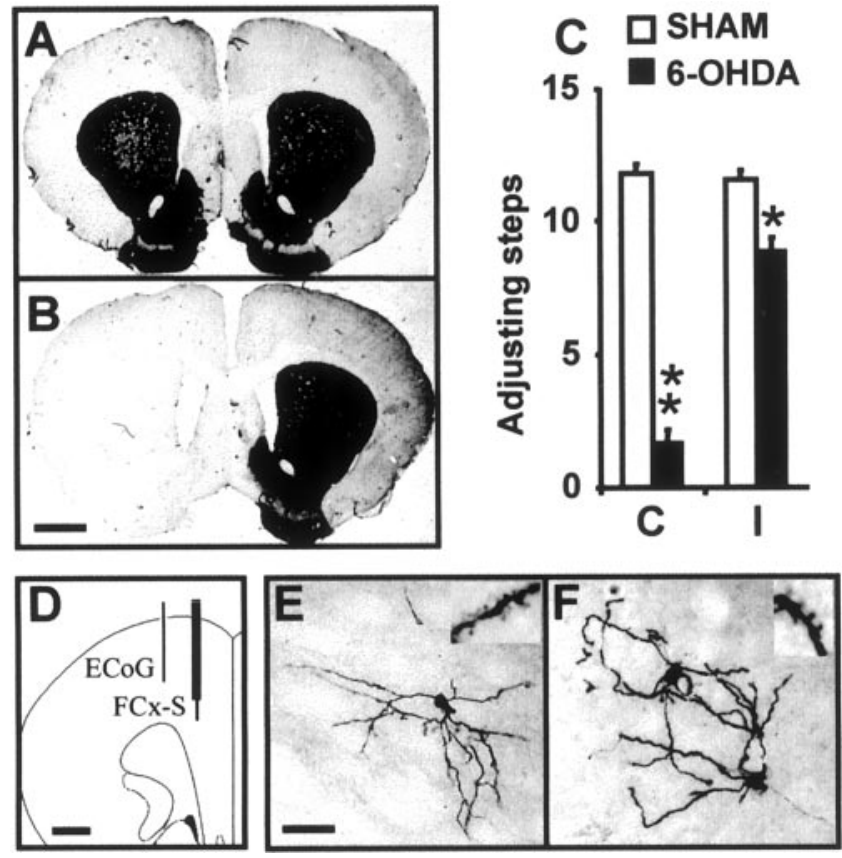

Figure 1. $A, B$, Coronal sections of the forebrain immunolabeled with antibodies directed against tyrosine hydroxylase. The microphotographs depict the typical aspect of the striatum in sham-lesioned $(A)$ and 6-OHDA-lesioned $(B)$ rats. Scale bar, $1.5 \mathrm{~mm}$. $C$, The behavioral effect of the lesion was evaluated with the "stepping test." The 6-OHDA-lesioned rats had a significant impairment in the test $(p<0.001$; main group effect in a two-way ANOVA) involving both the contralateral forelimb ( ${ }^{*} p<$ 0.05; Tukey's test) and, although in a smaller degree, the ipsilateral forelimb (* ${ }^{*}<0.05$; Tukey's test). $D$, Schematic diagram showing the placement of the concentric bipolar electrodes used to record the electrocorticogram (lateral electrode) and to stimulate the frontal cortex (medial electrode). Scale bar, $1.2 \mathrm{~mm}$. $E, F$, All the neurons that were successfully injected with Neurobiotin had the typical morphology of striatal medium spiny neurons. In sham-lesioned rats, all injections yielded a single labeled cell $(E)$, whereas in 6-OHDA-lesioned rats, $\sim 60 \%$ of the injections yielded two neurons $(F)$. Insets, Segments of dendrites showing spines. Scale bar, $25 \mu \mathrm{m}$. ECoG, Electrocorticogram; $F C x-S$, frontal cortex stimulation; $C$, contralateral; $I$, ipsilateral.

input resistance, firing threshold, and action potential duration and amplitude (Table 1).

Twenty-two neurons in the control group and 23 in the 6-OHDA-lesioned group showed a fluctuating membrane potential (Fig. 2). The membrane potential fluctuations differed between groups (Table 1) (Fig. 3). The voltage during both the down and up states was significantly more depolarized in 6-OHDA-lesioned rats than control rats. Considering that up events are perceived as enabling states during which MSNs can fire action potentials (Wilson, 1993; O’Donnell and Grace, 1995), the above described alterations probably underlie the increased firing rates reported for striatal single units in 6-OHDA-lesioned rats (Arbuthnott, 1974; Schultz, 1982; Nisenbaum et al., 1986). Actually, for the spontaneously active neurons, the probability of firing at least one action potential during an up state was significantly higher in 6-OHDA-lesioned than control rats (Fig. 4). Furthermore, striatal neurons from 6-OHDA-lesioned rats spent significantly less time in the down state and required more time to shift from one state to the other (Fig. 3). The contrast between the experimental groups is further emphasized by the finding that control rats had a significantly higher proportion of silent neurons than 6-OHDA-lesioned rats (Table 1). 
Table 1. Electrophysiological properties of intracellularly recorded striatal neurons in control and 6hydroxydopamine-lesioned rats

\begin{tabular}{|c|c|c|}
\hline & Control & 6-OHDA \\
\hline Number of silent neurons & $13^{a}$ & 4 \\
\hline Number of neurons firing spontaneously & 14 & 27 \\
\hline Action potential amplitude $(\mathrm{mV})^{b}$ & $50.3 \pm 1.8$ & $50.9 \pm 0.9$ \\
\hline Action potential duration $(\mathrm{msec})^{c}$ & $0.85 \pm 0.03$ & $0.81 \pm 0.03$ \\
\hline Threshold (spontaneous firing) $(\mathrm{mV})^{d}$ & $-54.8 \pm 1.0$ & $-54.5 \pm 0.8$ \\
\hline Input resistance $(\mathrm{M} \Omega)^{e}$ & $53.0 \pm 3.3$ & $51.9 \pm 4.7$ \\
\hline \multicolumn{3}{|l|}{ Silent neurons } \\
\hline Voltage in the down state $(\mathrm{mV})$ & $-87.4 \pm 3.5$ & $-87.4 \pm 4.3$ \\
\hline Voltage in the up state $(\mathrm{mV})$ & $-77.5 \pm 3.8$ & $-76.8 \pm 4.7$ \\
\hline \multicolumn{3}{|l|}{ Spontaneously active neurons } \\
\hline Voltage in the down state $(\mathrm{mV})$ & $-82.4 \pm 3.1$ & $-73.3 \pm 1.8^{f}$ \\
\hline Voltage in the up state $(\mathrm{mV})$ & $-69.7 \pm 2.1$ & $-63.1 \pm 1.3^{g}$ \\
\hline $\begin{array}{l}\text { Duration of the recordings (minutes, } \\
\text { mean } \pm \mathrm{SEM} \text {, and range })^{h}\end{array}$ & $49 \pm 8(11-179)$ & $51 \pm 9(11-208)$ \\
\hline
\end{tabular}

Data are mean \pm SEM. Spike measures were taken from spontaneously active neurons.

${ }^{a} p=0.004$ Fisher exact probability test.

${ }^{b}$ Measured from threshold.

${ }^{c}$ Measured at half maximal amplitude.

${ }^{d}$ Defined as the membrane potential at which the rate of voltage change exceeded $4 \mathrm{~V} / \mathrm{sec}$ (Wickens and Wilson, 1998).

${ }^{e}$ Measured by applying depolarizing current pulses during the down state.

${ }_{p} p=0.012$, two-tailed Student's $t$ test.

${ }^{g} p=0.046$, two-tailed Student's $t$ test.

${ }^{h}$ Includes the time spent in the application of Neurobiotin.

Spectral analysis of the membrane potential of bistable-like neurons revealed that $>90 \%$ of the spectral power was concentrated in the frequency range comprised between $0.4-2 \mathrm{~Hz}$ (Table 2; see Figs. 6 and 7). All two-state neurons had peaks within this frequency range that largely exceeded the $95 \%$ confidence interval of the mean relative spectral power. Note that for spontaneously active neurons the action potentials have been truncated before performing the FFT. Consequently, spectral profiles reflect true membrane potential frequency components. State transitions probably explain most of the spectral power within this frequency range (Stern et al., 1997). The frequency of state transitions, as estimated from counts performed on the same signal segments, was $0.95 \pm 0.05$ in control rats and $1.03 \pm 0.07$ in 6-OHDA-lesioned rats ( $p=0.34$, two-tailed $t$ test).

For five neurons in the control group and eight in the 6-OHDA-lesion group, it was not possible to ascertain the existence of a two-state membrane potential. The small number of this kind of recordings in our sample prevented the investigation of between groups differences.

\section{Response of striatal neurons to cortical stimulation}

As reported previously (for review, see Wilson, 1993), cortical stimulation evoked a short latency dPSP, followed by a sequence consisting of a prolonged hyperpolarization and a plateau depo-

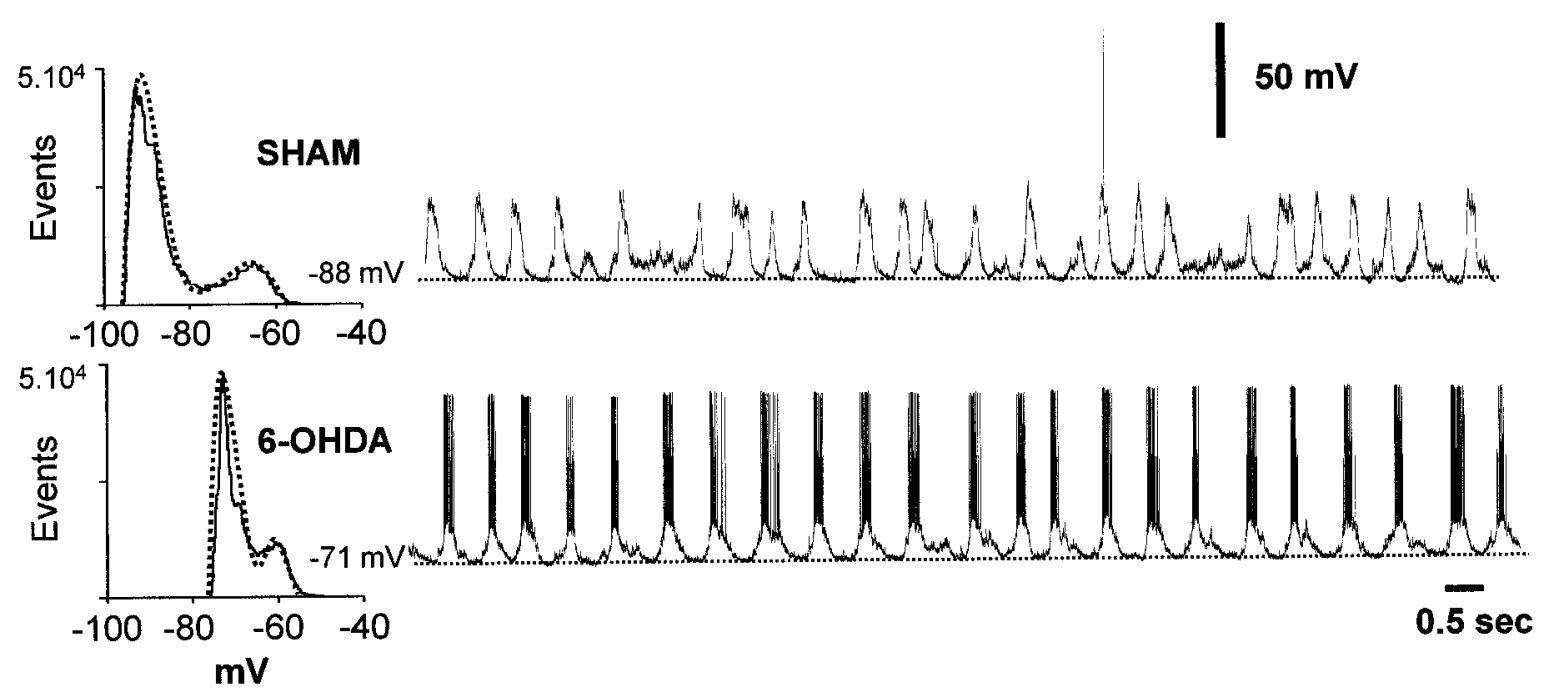

Figure 2. Representative recordings of striatal neurons from sham-lesioned rats (top trace) and 6-OHDA-lesioned rats (bottom trace). In both experimental groups, most neurons had a fluctuating membrane potential. Histograms depicting the time spent at any given membrane potential typically had bimodal profiles with the distributions fitting dual-Gaussian functions. 

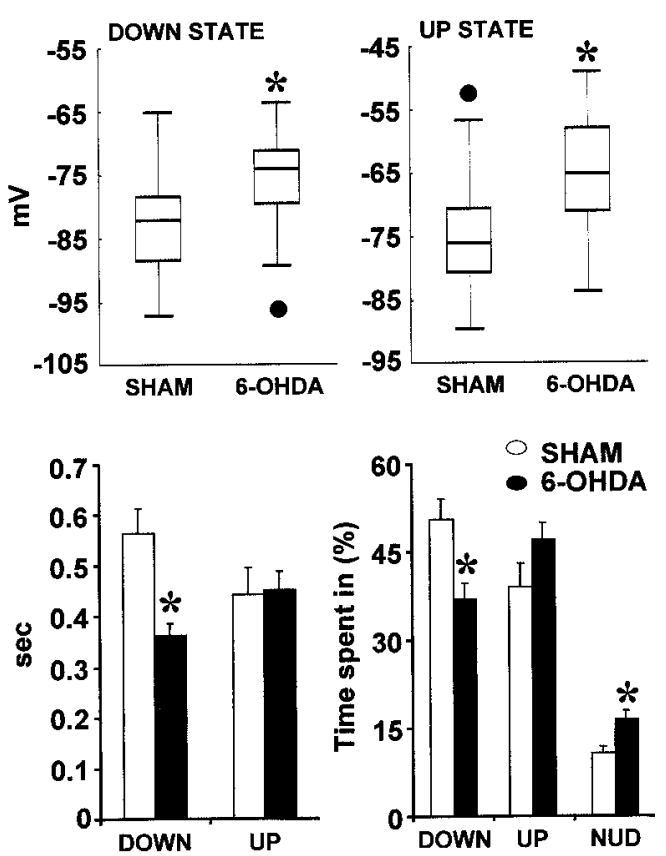

Figure 3. Sham-lesioned and 6-OHDA-lesioned rats differed in several measurements related to their two-state membrane potential. Top, The membrane potential was more depolarized during both the down states $\left({ }^{*} p=0.027\right)$ and up states $\left({ }^{*} p=0.004\right.$; two-tailed Student's $t$ test $)$ in rats with nigrostriatal lesions. The box-plots include data from both silent and active neurons. Median (black line), 25th and 75th percentiles (bar limits), 10 th and 90th percentiles (error bars), and outliers (black circles) are shown. Bottom left, Down states were shorter in 6-OHDA-lesioned rats $\left({ }^{*} p<0.001\right.$; two-tailed Student's $t$ test). The duration of the up events was not significantly different between groups. Bottom right, In a given time window, neurons from 6-OHDA-lesioned rats spent a smaller proportion of time in the down state $\left({ }^{*} p=0.0044\right.$; ANOVA by ranks) and a higher proportion of time shifting from one state to the next (fluctuating around the transitional voltage or reaching short-lived steady states) (NUD) $\left({ }^{*} p=\right.$ 0.011 , ANOVA by ranks). There was a trend toward an increased proportion of time spent in the up state that did not reach statistical significance $\left({ }^{*} p=0.10\right.$; ANOVA by ranks).

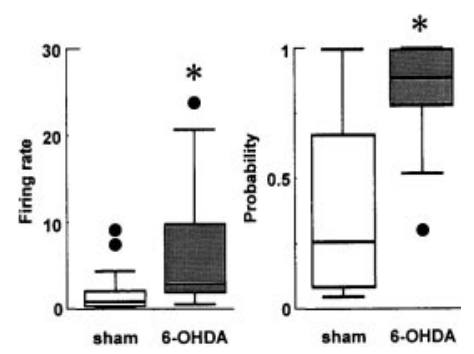

Figure 4. Box-plots summarizing information regarding the firing rate and firing probability of striatal neurons. Median (black line), 25th and 75th percentiles (bar limits), 10th and 90th percentiles (error bars), and outliers (black circles) are shown. Left, Seventy-five percent of the spontaneously active neurons recorded from 6-OHDA-lesioned rats had firing rates $>3 \mathrm{~Hz}$, whereas $75 \%$ of the spontaneously active neurons recorded from control rats had rates $<3 \mathrm{~Hz}\left({ }^{*} p=0.002\right.$; Mann-Whitney $U$ test). Right, The spontaneously active neurons recorded from control rats had a significantly lower probability of firing at least one spike during an up event than those recorded from rats with nigrostriatal lesions $\left({ }^{*} p=0.011\right.$; Mann-Whitney $U$ test).

larization. The latter sequence closely resembled the spontaneous membrane potential shifts of striatal neurons (Fig. 5). Although the short latency dPSP is probably produced by activation of a small volume of cortical tissue around the electrode tip, the
Table 2. Characterization of the response of intracellularly recorded striatal neurons to cortical stimulation in control and 6hydroxydopamine-lesioned rats

\begin{tabular}{lcc} 
& Control & 6-OHDA \\
\hline Fast dPSP & & \\
$\quad$ Time to the peak (msec) & $20.6 \pm 0.7$ & $21.7 \pm 0.9$ \\
$\quad$ Amplitude (mV) & $16.4 \pm 1.6$ & $15.0 \pm 2.3$ \\
Long-lasting hyperpolarization & & \\
$\quad$ Duration (msec) & $415.1 \pm 30.3$ & $293.2 \pm 16.8^{a}$ \\
$\quad$ Latency (msec) & $58.1 \pm 4.5$ & $64.1 \pm 7.3$ \\
$\quad$ Membrane potential in & & \\
$\quad$ steady-state (mV) & $-91.8 \pm 1.9$ & $-83.8 \pm 2.3^{b}$ \\
Long-lasting depolarization & & \\
$\quad \begin{array}{l}\text { Duration (msec) } \\
\text { Latency (msec) }\end{array}$ & $521.7 \pm 97.1$ & $555.8 \pm 138.7$ \\
$\quad$ Membrane potential in steady & $466.9 \pm 30.3$ & $352.6 \pm 16.9^{c}$ \\
$\quad$ state (mV) & $-76.2 \pm 1.9$ & $-71.7 \pm 1.9^{d}$ \\
\hline
\end{tabular}

Data are mean \pm SEM of $n=8-20$ neuronal responses. The responses were evoked by single pulse cortical stimulation $(400 \mu \mathrm{A}$ square wave pulses at $0.5 \mathrm{~Hz})$.

${ }^{a} p=0.0007$, two-tailed Student's $t$ test.

${ }^{b} p=0.019$, two-tailed Student's $t$ test.

${ }^{c} p=0.0013$, two-tailed Student's $t$ test.

${ }^{d}$ NS $(p=0.102)$, two-tailed Student's $t$ test.
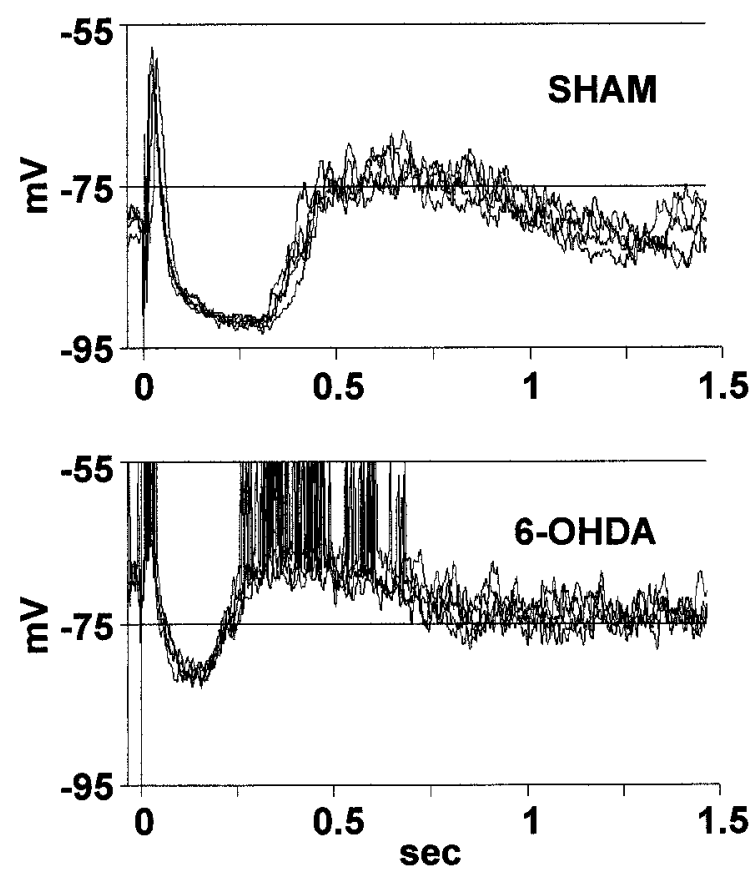

Figure 5. Typical examples of the response evoked by cortical stimulation in sham-lesioned (top) and 6-OHDA-lesioned rats (bottom). Several events were superimposed in each graphic. Note the shorter latency of the depolarizing plateau and the more depolarized membrane potential during the hyperpolarizing phase of the response in the 6-OHDAlesioned rats.

hyperpolarization-depolarization sequence is probably driven by a hypersynchronous cortical oscillatory cycle that indicates resumption of the endogenously generated rhythm (Wilson, 1993). After a single pulse applied to a cortical area, neighbor cortical neurons display a short latency dPSP, followed by a long-lasting inhibition and a postinhibitory depolarizing plateau (Amzica and Steriade, 1998); the shape and time course of this response closely resembles that of striatal neurons. 
The shape of the response of striatal neurons to cortical stimulation, time to the peak of the fast dPSP, and peak amplitude of the fast dPSP were similar in both experimental groups (Table 2). In agreement with the results reported above regarding naturally occurring state transitions in the signal, the long-lasting hyperpolarization was shorter and had a more depolarized potential in 6-OHDA-lesioned rats than in control rats (Fig. 5). The plateau depolarization had a significantly reduced latency and reached a slightly more depolarized potential (the difference between groups was not significant) in 6-OHDA-lesioned rats (Table 2).

\section{A low-frequency oscillation is encoded in spike trains of striatal neurons}

Most striatal neurons show very low mean firing rates (many are silent) in control rats, but in 6-OHDA-lesioned rats their firing rates are significantly increased (Arbuthnott, 1974; Schultz, 1982; Nisenbaum et al., 1986) (Fig. 4). Because striatal neurons fire action potentials during the up states, and the probability of firing at least one spike during an up state is increased in 6-OHDAlesioned rats, it seemed likely that the slow membrane potential fluctuation would be transmitted more effectively to striatal target nuclei in rats with nigrostriatal lesions. To provide evidence in support of this hypothesis, we sought for low-frequency oscillations in spike trains of striatal neurons recorded from control and 6-OHDA-lesioned rats. Only those cells having firing rates higher than 1 spike per second were analyzed (Fig. 4). A significant $\sim 1$ $\mathrm{Hz}$ periodic frequency component could be retrieved from 4 of 22 control rat recordings ( $14.8 \%$ of the sampled neurons) and from 15 of $236-O H D A-l e s i o n e d$ rat recordings (67.7\% of the sample) $(p=0.0023$, Fisher exact test) (see Fig. 7).

\section{Correlation between the ECoG and the membrane potential of striatal neurons}

Intracellular recordings of two-state striatal neurons were obtained simultaneously with the frontal cortex ECoG in 14 instances from 12 control rats, and in 16 instances from 13 6-OHDA-lesioned rats. For 13 signal pairs in the control group and 12 in the 6-OHDA-lesioned group, clear signs of synchronized oscillation were found. In both experimental groups, the power spectra of the signals revealed that the ECoG and the membrane potential of the simultaneously recorded striatal neuron had the highest relative power at a closely similar frequency, usually in the $0.4-2 \mathrm{~Hz}$ range (Figs. 6,7 ). The signals had a very strong coherence at the dominant spectral frequency, suggesting that the waveforms were oscillating synchronously. The experimental groups did not differ in the mean coherence at the dominant frequency or in the phase relationship between the ECoG and intracellular recording (Table 3). Phase lags, estimated at the coherent frequency, ranged from -155 to $+72 \mathrm{msec}$ (negative means that the ECoG precedes the striatal recording), with the ECoG anteceding the intracellular signal in 15 of 25 instances (Table 3). Similarly, the cross-correlograms revealed a strong correlation between the ECoG and membrane potential fluctuations of striatal neurons (Fig. 6). Lags between the signals, estimated from the cross-correlograms, were consistent with those calculated from the phase spectra (Table 3). The close relationship between the signals was consistently retained over time, as indicated by the analysis of several nonoverlapping $30 \mathrm{sec}$ epochs within time windows spanning several minutes (Fig. 6). For the remaining five signal pairs, a close relationship between the ECoG and intracellular recording could not be evidenced, i.e., they correlated weakly and showed low coherence.

\section{DISCUSSION}

Membrane potential fluctuations of striatal neurons are correlated with slow oscillatory activity in the frontal cortex electrocorticogram

In slow wave sleep and anesthesia, the EEG of animals and humans displays synchronized oscillations of different frequencies. A slow oscillation $(\sim 1 \mathrm{~Hz})$, which is generated intracortically, seems to be responsible for the grouping of delta waves and spindles, and is a major determinant of the shape of the EEG waveform (Steriade, 1999). In vivo intracellular recordings revealed that the membrane potential of cortical neurons shows rhythmic depolarizing and hyperpolarizing shifts that are strongly correlated with the EEG slow oscillatory activity (Amzica and Steriade, 1995). During the depolarizing phase of the membrane potential fluctuation, cortical neurons discharge bursts of action potentials that grant spreading of the rhythm through the cortex and thalamus (Steriade 1999). Our simultaneous recordings of striatal neurons and the frontal ECoG indicate that membrane potential fluctuations reflect spreading of cortical slow rhythms to the striatum. This conclusion is supported by previous findings indicating that up states in striatal neurons are driven by inputs from the cerebral cortex: (1) up states are abolished by decortication (Wilson 1993); (2) striatal neurons do not show up events in slices, but display up states in chronic organotypic cortex-striatum cocultures (Plenz and Aertsen, 1996); (3) corticostriatal neurons display membrane potential fluctuations that resemble those of striatal neurons in many aspects, including the presence of common low-frequency components in the power spectra (Stern et al., 1997). Studies on the origin of state transitions in nucleus accumbens spiny neurons are consistent with our results. In the nucleus accumbens, up events are driven by excitatory hippocampal input (O'Donnell and Grace, 1995), and membrane potential fluctuations are correlated with shifts in hippocampal field potential (Goto and O'Donnell, 2001). Finally, a report by Mahon et al. (2001) indicates that slow waves in the EEG are correlated with depolarizing states both in corticostriatal and striatal neurons under a variety of anesthetic conditions. It is likely that spreading of slow cortical rhythms to the striatum occurs via the massive corticostriatal pathway. In the present study, the mean time lag between cortical and striatal signals was similar to the latency of the fast dPSP that was evoked in striatal neurons by cortical stimulation.

\section{Chronic nigrostriatal lesions alter the membrane potential of striatal neurons, allowing transmission of slow cortical oscillations to the basal ganglia output nuclei}

Spreading of slow cortical rhythms to striatal target nuclei seems to be limited in healthy animals. Neurons of the GP and SNpr display tonic regular firing during natural slow wave sleep (Datta et al., 1991; Urbain et al., 2000) and anesthesia (Pan and Walters, 1988; MacLeod et al., 1990; Murer et al., 1997; Ni et al., 2000). After chronic nigrostriatal lesions, SNpr units display rhythmic burst firing (MacLeod et al., 1990; Tseng et al., 2000) that is strongly modulated by intrastriatal administration of DA receptor agonists (Murer et al., 1997; Tseng et al., 2000). The capability of MSNs to transmit slow cortical rhythms to their target nuclei is constrained by their very low firing probability in control animals. Our results suggest that after chronic nigrostriatal lesions, an increased excitability of striatal neurons allows transmission of slow cortical rhythms to striatal target nuclei (Figs. 7, 8).

Changes in the shape of the fluctuating membrane potential 


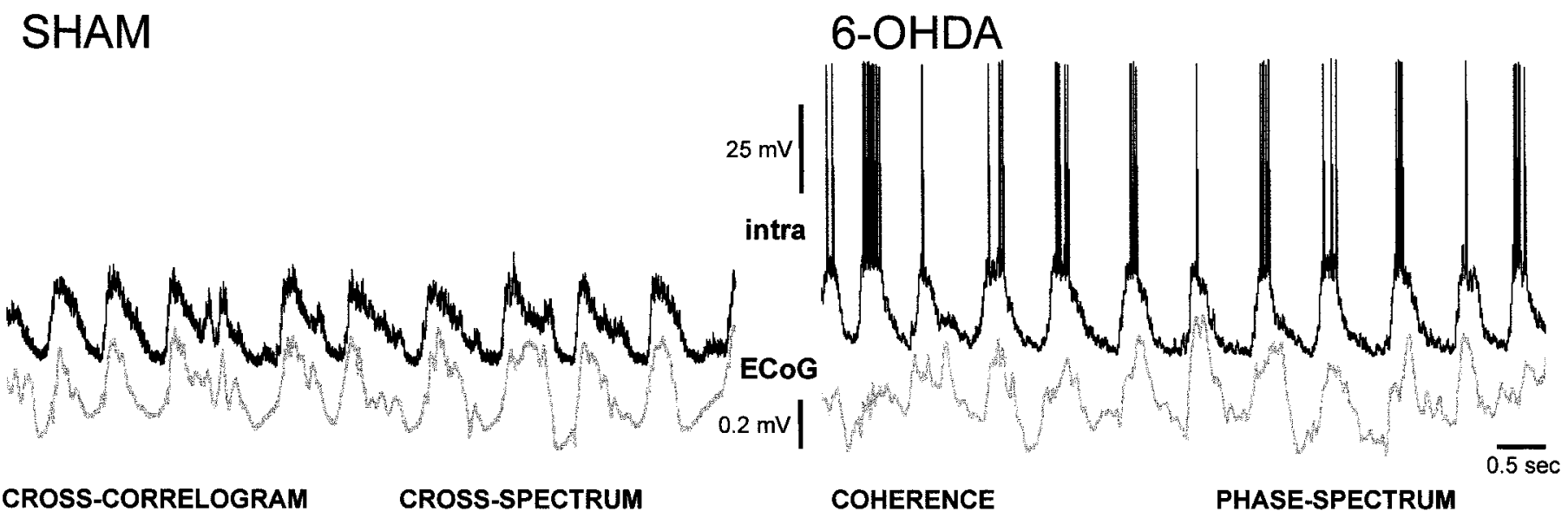

\section{SHAM}
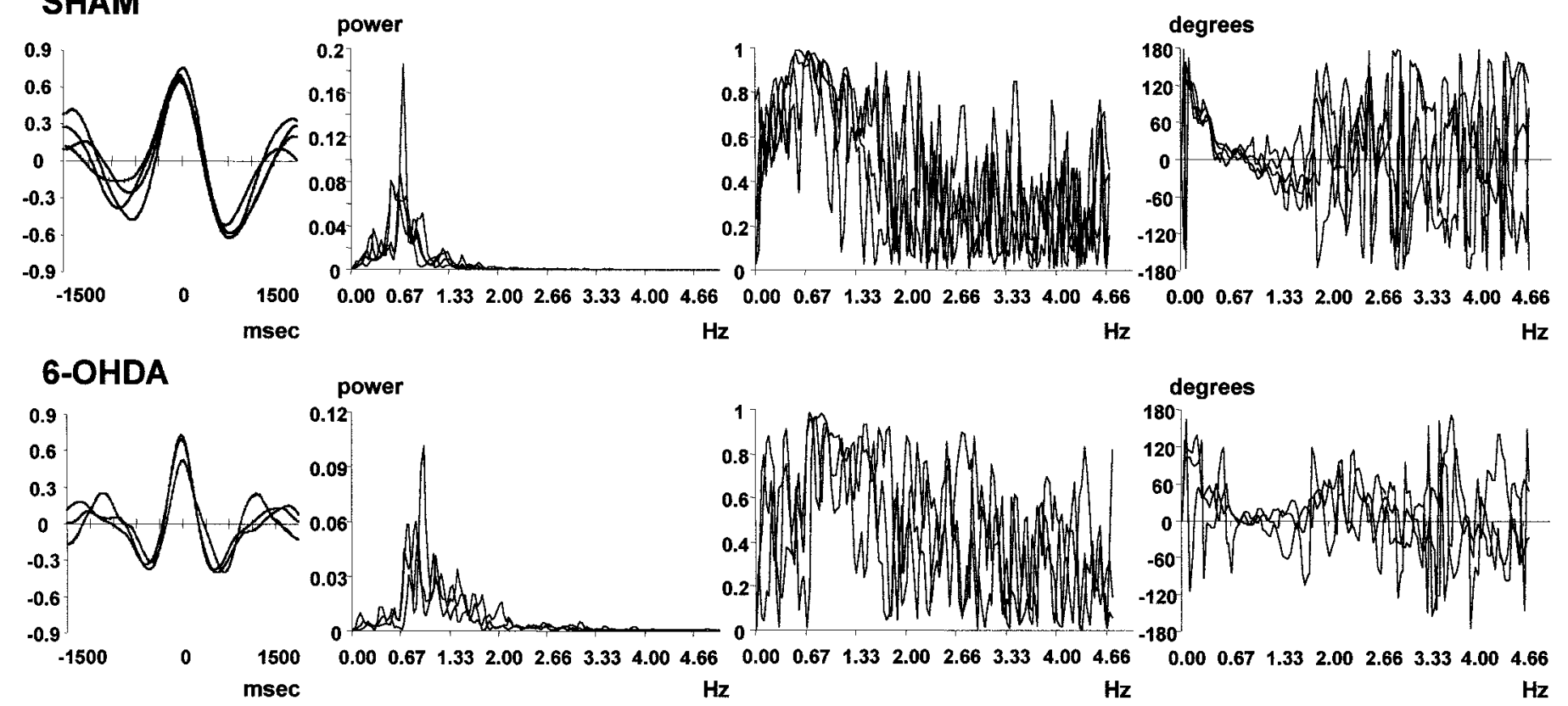

Figure 6. Visual inspection of simultaneous recordings of the frontal electrocorticogram and the membrane potential of striatal neurons indicated that the two waveforms were oscillating synchronously at $\sim 1 \mathrm{~Hz}$. The signals are displayed as they were recorded, but note that they were down-sampled, smoothed, and standardized before analysis. The belief that the signals were synchronized was substantiated through the analysis of cross-correlograms and by means of coherence analysis. Each row of graphics shows the cross-correlogram, cross-spectrum, coherence spectrum, and phase-spectrum corresponding to the signal pairs displayed in the upper part of the figure (top row of graphics, sham-lesioned rat; bottom row of graphics, rat with nigrostriatal lesion). In each graphic, the results of the analysis of several $30 \mathrm{sec}$ epochs from the same signal pair were superimposed. The four disjoined $30 \mathrm{sec}$ epochs depicted for the sham-lesioned rat were chosen from a recording session elapsing 15 min. A 12 min recording session from a 6-OHDA-lesioned rat provided the three $30 \mathrm{sec}$ epochs that were chosen for the bottom row of graphics. Both signal pairs showed strong correlations. The cross-spectra revealed a powerful common frequency component of $\sim 0.7 \mathrm{~Hz}(S H A M)$ or $\sim 0.9 \mathrm{~Hz}(6-O H D A)$, and the signals displayed a very high coherence and a lineal phase relationship at the dominant frequency.

seem to underlie the increased firing probability of striatal neurons in 6-OHDA-lesioned rats. Striatal neurons recorded from rats with nigrostriatal lesions displayed a more depolarized membrane potential, during both the down and up states, and spent less time in the down state. The mechanisms leading to these changes remain to be determined. Current knowledge suggests that they may be multiple and complex. Dopamine increases potassium currents that keep the resting potential near the potassium equilibrium potential (Pacheco-Cano et al., 1996; Waszczak et al., 1998). A reduced activity of these currents may partly explain the more depolarized membrane potential and shorter duration of the down state in rats with nigrostriatal lesions. Dopamine receptors also modulate in a complex way currents that are active within the voltage range reached during the up events. For example, D2 receptor stimulation suppresses currents through L-type calcium channels and consequently, reduces striatal neuron excitability (Hernández-López et al., 2000). There is in vivo evidence demonstrating that L-type calcium channel blockers reduce glutamate-induced bursts in nucleus accumbens neurons (Cooper and White, 2000). Increased currents through L-type calcium channels may have yielded to a more depolarized potential during the up states, and an increased firing probability, in rats with chronic nigrostriatal lesions. In addition, in vitro evidence supports that corticostriatal transmission is increased in slices from 6-OHDA-lesioned rats. Striatal neurons do not show spontaneous firing in control or in DA-depleted slices, but the 


\section{SHAM}

ECOG

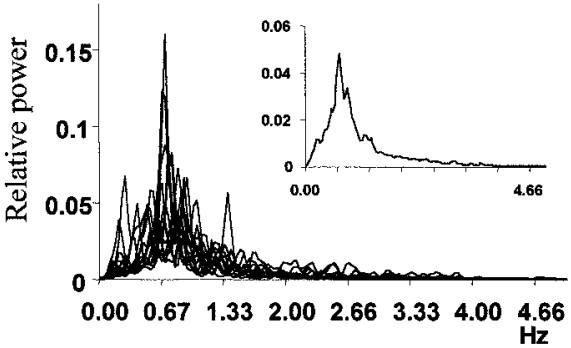

Vm

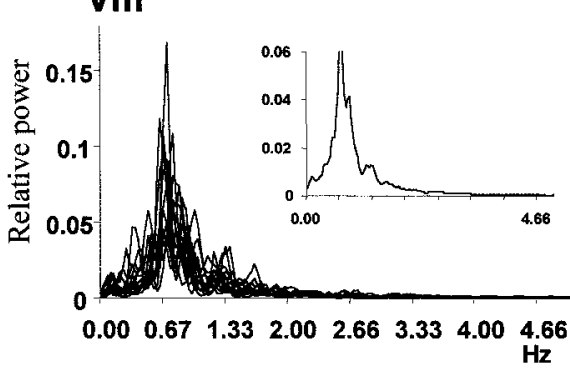

Spike trains

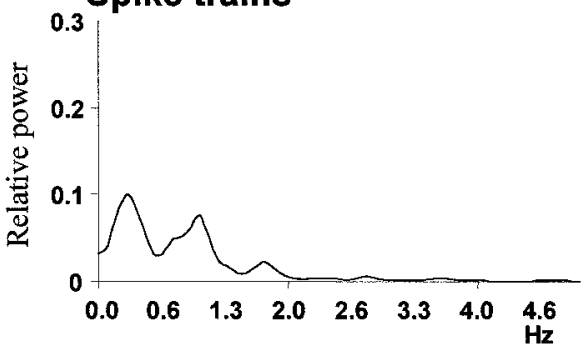

\section{6-OHDA}
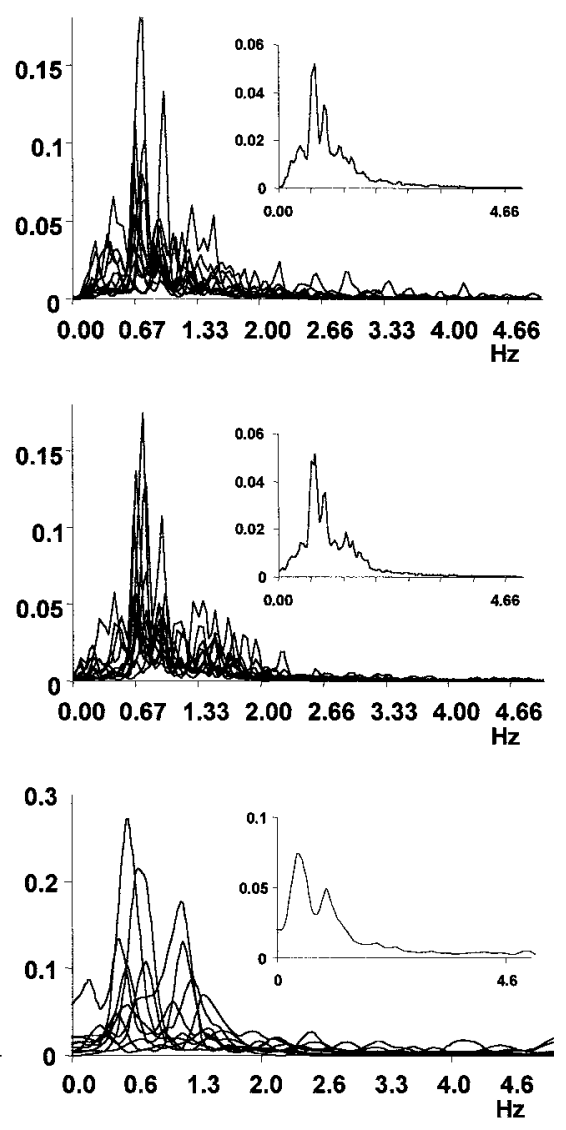

Figure 7. Each column depicts the power spectra of the electrocorticogram $(E C o G)$, membrane potential $(\mathrm{Vm})$, and spike trains of all the well correlated signal pairs recorded from sham $(n=13)$ and 6-OHDAlesioned $(n=12)$ rats. The insets are averages of all the spectra contained in the corresponding graphic. Only one neuron in the pairs recorded from shamlesioned rats displayed enough spontaneous discharge $(>1 \mathrm{~Hz})$ to compute its spike train spectrum. For 6-OHDA-lesioned rats, $\sim 70 \%$ of these recordings showed firing rates higher than $\sim 1 \mathrm{~Hz}$.

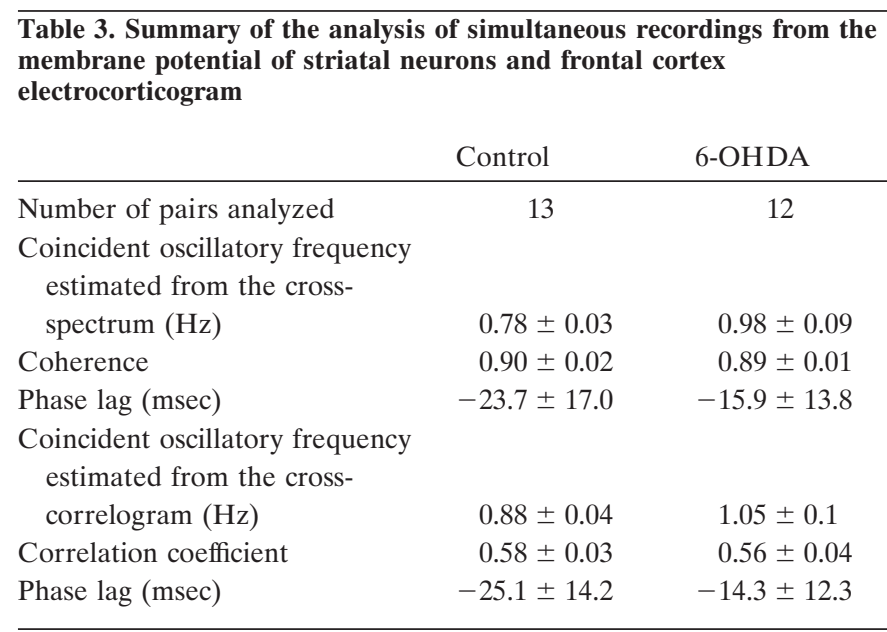

Data are mean \pm SEM. There were not significant differences between groups. Phase lags were expressed with the same sign for the estimations performed from cross-correlograms and phase spectra to facilitate comparisons. Negative implies that the ECoG preceded the intracellular recording.

number and amplitude of spontaneous dPSPs is increased in the DA-denervated striatum (Galarraga et al., 1987; Calabresi et al., 1993). Both presynaptic and postsynaptic mechanisms (Brown and Arbuthnott, 1983; Cepeda et al., 1993; Umemiya and Raymond, 1997) may account for the increase in spontaneous corticostriatal transmission after chronic nigrostriatal lesions.

It may be argued that an increased susceptibility to damage induced by the intracellular recording electrode might have pro- duced the differences in striatal neuronal activity between shamand 6-OHDA-lesioned rats. This possibility seems unlikely, however, because: (1) reports based on extracellular single unit recordings have demonstrated an increased firing rate of striatal units in 6-OHDA-lesioned rats (Arbuthnott, 1974; Schultz, 1982; Nisenbaum et al., 1986); (2) action potential amplitude and duration, input resistance, and the duration of recordings were not different between control and 6-OHDA-lesioned rats (Table 1); (3) preliminary results indicate that the changes in membrane potential induced by 6-OHDA lesions can be reversed by systemic administration of dopamine receptor agonists, but not by vehicle injections (our unpublished observations).

\section{Multiple pathways may be involved in the transmission of slow cortical rhythms to the globus pallidus and basal ganglia output nuclei}

The striatum is probably not the only structure involved in transmission of slow cortical rhythms to the remaining basal ganglia. Recent work by Magill et al. (2000) demonstrated that slow cortical rhythms are reflected in spike trains of subthalamic nucleus (STN) and GP neurons in ketamine-anesthetized nonlesioned rats and suggested that cortical rhythms are propagated via cortico-STN-GP connections. Most GP neurons show tonic regular firing in natural slow wave sleep (Urbain et al., 2000), as well as under the influence of other anesthetics (Pan and Walters, 1988; Magill et al., 2000; Ni et al., 2000) and in paralyzed animals (Pan and Walters, 1988), however, suggesting that ketamine may propitiate the propagation of slow cortical rhythms through the basal ganglia. After chronic nigrostriatal lesions, GP neurons 

of rhythmic modulations of firing rate in the basal ganglia output nuclei. The $\sim 1 \mathrm{~Hz}$ cortical rhythm is propagated to the striatum, where it produces a striatal projection neurons. During the depolarizing phase of the membrane potential fluctuation, striatal neurons are very close to threshold, but they only rarely discharge action potentials when the nigrostriatal system is intact (left). In animals having chronic nigrostriatal lesions, striatal projection neurons show a more depolarized up state and an increased firing probability (right). These changes may allow transmission of a coarse representation of the cortical rhythm to the striatal target nuclei.
Figure 8. Postulated mechanism for the generation rhythmic fluctuation in the membrane potential of

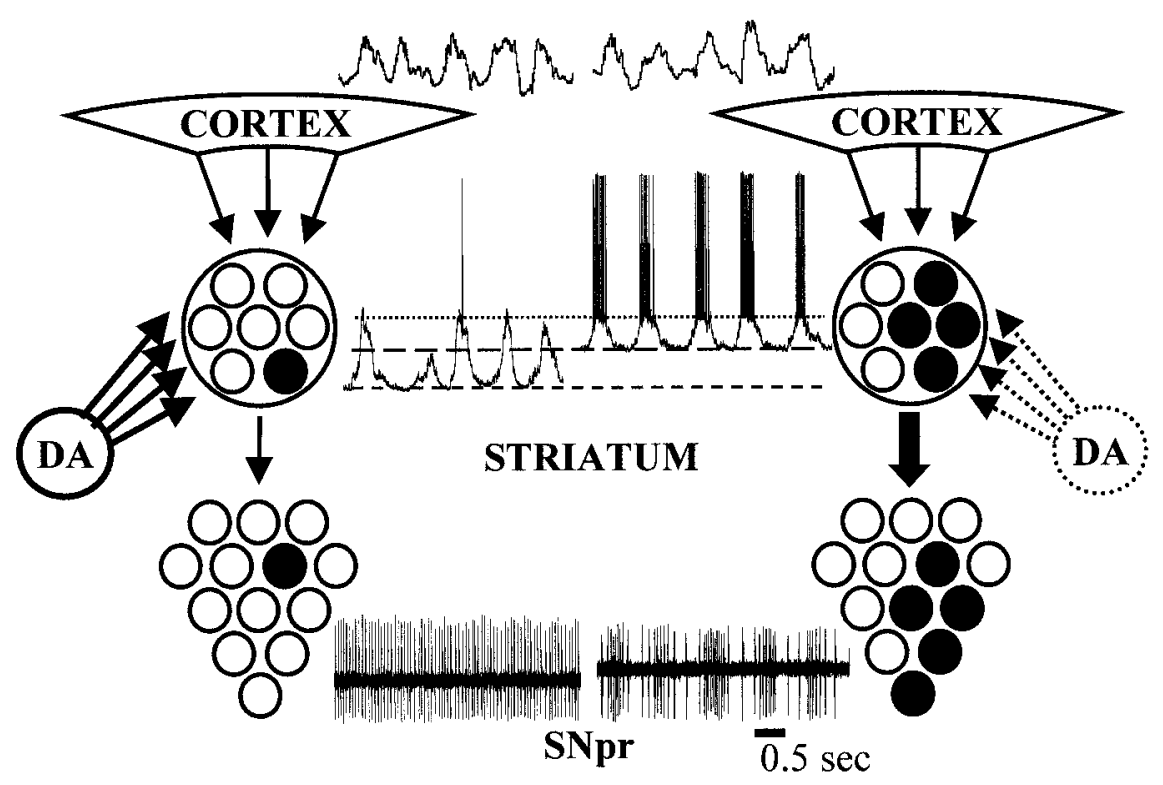

depolarized (enabling) state. We are not aware of studies aimed at characterizing the oscillatory behavior of basal ganglia neurons in parkinsonian monkeys during slow wave sleep, nor those of awake 6-OHDA-lesioned rats. Undoubtedly, this issue will be clarified in the near future. proportion of SNpr units displaying rhythmic bursting is reduced to half (Tseng et al., 2000), and rhythmic bursting in the GP is almost abolished (Ni et al., 2000) after STN lesions in 6-OHDAlesioned rats.

\section{Implications for Parkinson's disease}

In the awake monkey, basal ganglia output nuclei neurons display nonoscillatory firing patterns. However, after methyl-phenyltetrahydropyridine-induced lesions, a significant proportion of units in the STN and basal ganglia output nuclei exhibit an oscillatory pattern of action potential firing (Bergman et al., 1994; Nini et al., 1995; Wichmann et al., 1999; Raz et al., 2000). It is becoming increasingly evident that a substantial proportion of units in the STN and GP exhibit oscillatory firing in individuals with Parkinson's disease (Hurtado et al., 1999; Levy et al. 2000; Magnin et al., 2000). The oscillatory basal ganglia output is supposed to give rise to tremor, one of the cardinal signs of the illness. Furthermore, it has been postulated that an oscillatory basal ganglia output may disrupt cortical information processing by promoting the recruitment of thalamocortical circuits in rhythmic firing patterns (Brown and Marsden, 1998). Although the phenomenon resembles what happens in rats after 6-OHDAinduced lesions, the main frequency of oscillatory activity in behaving parkinsonian primates $(3-20 \mathrm{~Hz})$ is higher than that found in anesthetized 6-OHDA-lesioned rats $(0.4-2 \mathrm{~Hz})$. If abnormal spreading of cortical slow rhythms through the striatum and STN underlies the rhythmic firing pattern of output nuclei neurons in parkinsonism, the different oscillatory periods observed in awake parkinsonian primates and anesthetized 6-OHDA-lesioned rats may merely reflect the distinct dominant EEG frequencies that characterize each behavioral state. It seems possible that a population of more excitable striatal neurons will be prone to transfer high-frequency cortical rhythms (like those observed in the cortex during quiet waking) to striatal targets. The reduced latency of the plateau depolarization that was evoked by cortical stimulation in 6-OHDA-lesioned rats suggests that more excitable striatal neurons can be driven easily to a

\section{REFERENCES}

Albin RL, Young AB, Penney JB (1989) The functional anatomy of basal ganglia disorders. Trends Neurosci 12:366-375.

Amzica F, Steriade M (1995) Short- and long-range neuronal synchronization of the slow $(<1 \mathrm{~Hz})$ cortical oscillation. J Neurophysiol 73:604-622.

Amzica F, Steriade M (1998) Cellular substrates and laminar profile of sleep K-complex. Neuroscience 82:671-686. tum after unilateral destruction of the dopamine input. J Physiol (Lond) 239:121P-122P.

Bergman H, Wichmann T, Karmon B, DeLong MR (1994) The primate subthalamic nucleus. II. Neuronal activity in the MPTP model of parkinsonism. J Neurophysiol 72:507-520.

Brown JR, Arbuthnott GW (1983) The electrophysiology of dopamine (D2) receptors: a study of the actions of dopamine on corticostriatal transmission. Neuroscience 10:349-355.

Brown P, Marsden CD (1998) What do the basal ganglia do? Lancet 351:1801-1804.

Calabresi P, Mercuri NB, Stefani A, Bernardi G (1990) Synaptic and intrinsic control of membrane excitability of neostriatal neurons. I. An in vivo analysis. J Neurophysiol 6:651-662.

Calabresi P, Mercuri NB, Sancesario G, Bernardi G (1993) Electrophysiology of dopamine-denervated striatal neurons. Brain 116:433-452.

Cepeda C, Walsh JP, Hull CD, Howard SG, Buchwald NA, Levine MS (1989) Dye-coupling in the neostriatum of the rat. I. Modulation by dopamine-depleting lesions. Synapse 4:229-237.

Cepeda C, Buchwald NA, Levine MS (1993) Neuromodulatory actions of dopamine in the neostriatum are dependent upon the excitatory amino acid receptor subtypes activated. Proc Natl Acad Sci USA 90:9576-9580.

Chang JW, Wachtel SR, Young D, Kang UJ (1999) Biochemical and anatomical characterization of forepaw adjusting steps in rat models of Parkinson's disease: studies on medial forebrain bundle and striatal lesions. Neuroscience 88:617-628.

Cooper DC, White F (2000) L-type calcium channels modulate glutamate-driven bursting activity in the nucleus accumbens in vivo. Brain Res 880:212-218.

Datta S, Curro Dossi R, Pare D, Oakson G, Steriade M (1991) Substantia nigra reticulata neurons during sleep-waking states: relation with ponto-geniculo-occipital waves. Brain Res 566:344-347.

Galarraga E, Bargas J, Martinez-Fong D, Aceves J (1987) Spontaneous synaptic potentials in dopamine-denervated neostriatal neurons. Neurosci Lett 81:351-355.

Goto Y, O'Donnell P (2001) Synchronous activity in the hippocampus and nucleus accumbens in vivo. J Neurosci 21:RC131(1-5).

Hernández-López S, Tkatch T, Perez-Garci E, Galarraga E, Bargas J,
Arbuthnott GW (1974) Spontaneous activity of single units in the stria- 
Hamm H, Surmeier DJ (2000) D2 dopamine receptors in striatal medium spiny neurons reduce L-type $\mathrm{Ca}^{2+}$ currents and excitability via a novel PLC $\beta 1$-IP3-calcineurin-signaling cascade. J Neurosci 20:8987-8995.

Hollerman JR, Tremblay L, Schultz W (2000) Involvement of basal ganglia and orbitofrontal cortex in goal-directed behavior. Prog Brain Res 126:193-215.

Hurtado JM, Gray CM, Tamas LB, Sigvardt KA (1999) Dynamics of tremor-related oscillations in the human globus pallidus: a single case study. Proc Natl Acad Sci USA 96:1674-1679.

Kita H, Armstrong W (1991) A biotin-containing compound N-(2aminoethyl)biotinamide for intracellular labeling and neuronal tracing studies: comparison with biocytin. J Neurosci Methods 37:141-150.

Levy R, Hutchison WD, Lozano A, Dostrovsky JO (2000) Highfrequency synchronization of neuronal activity in the subthalamic nucleus of parkinsonian patients with limb tremor. J Neurosci 20:7766-7775.

Lopes da Silva F, Pijn JP, Boeijinga P (1989) Interdependence of EEG signals: linear vs. non-linear associations and the significance of time delays and phase shifts. Brain Topogr 2:9-18.

MacLeod NK, Ryman GW, Arbuthnott GW (1990) Electrophysiological properties of nigrothalamic neurons after 6-hydroxydopamine lesions in the rat. Neuroscience 38:447-456.

Magill PJ, Bolam JP, Bevan MD (2000) Relationship of activity in the subthalamic nucleus-globus pallidus network to cortical electroencephalogram. J Neurosci 20:820-833.

Magnin M, Morel A, Jeanmonod D (2000) Single-unit analysis of the pallidum, thalamus and subthalamic nucleus in parkinsonian patients. Neuroscience 96:549-564.

Mahon S, Deniau JM, Charpier S (2001) Relationship between EEG potentials and intracellular activity of striatal and cortico-striatal neurons. An in vivo study under different anesthetics. Cereb Cortex 11:360-373.

Murer MG, Riquelme LA, Tseng KY, Pazo JH (1997) Substantia nigra pars reticulata single unit activity in normal and 6-OHDA-lesioned rats: effects of intrastriatal apomorphine and subthalamic lesions. Synapse 27:278-293.

Ni Z, Bouali-Benazzouz R, Gao D, Benabid AL, Benazzouz A (2000) Changes in the firing pattern of globus pallidus neurons after the degeneration of nigrostriatal pathway are mediated by the subthalamic nucleus in the rat. Eur J Neurosci 12:4338-4344.

Nicola SM, Surmeier J, Malenka RC (2000) Dopaminergic modulation of neuronal excitability in the striatum and nucleus accumbens. Annu Rev Neurosci 23:185-215.

Nini A, Feingold A, Slovin H, Bergman H (1995) Neurons in the globus pallidus do not show correlated activity in the normal monkey, but phase-locked oscillations appear in the MPTP model of parkinsonism. J Neurophysiol 74:1800-1805.

Nisenbaum ES, Stricker EM, Zigmond MJ, Berger TW (1986) Longterm effects of dopamine-depleting brain lesions on spontaneous activity of type II striatal neurons: relation to behavioral recovery. Brain Res 398:221-230.

O'Donnell P, Grace AA (1995) Synaptic interactions among excitatory afferents to nucleus accumbens neurons: hippocampal gating of prefrontal cortical input. J Neurosci 15:3622-3639.

Olsson M, Nikkhah G, Bentlage C, Bjorklund A (1995) Forelimb akinesia in the rat Parkinson model: differential effects of dopamine agonists and nigral transplants as assessed by a new stepping test. J Neurosci $15: 3863-3875$.

Onn SP, Grace AA (1999) Alterations in electrophysiological activity and dye coupling of striatal spiny and aspiny neurons in dopamine denervated rat striatum recorded in vivo. Synapse 33:1-15.

Pacheco-Cano MT, Bargas J, Hernández-López S, Tapia D, Galarraga E
(1996) Inhibitory action of dopamine involves a subthreshold Cs+ sensitive conductance in neostriatal neurons. Exp Brain Res 110:205-211.

Pan HS, Walters JR (1988) Unilateral lesion of the nigrostriatal pathway decreases the firing rate and alters the firing pattern of globus pallidus neurons in the rat. Synapse 2:650-656.

Paxinos G, Watson C (1997) The rat brain in stereotaxic coordinates, Ed 3. London: Academic.

Plenz D, Aertsen A (1996) Neural dynamics in cortex-striatum cocultures. II. Spatiotemporal characteristics of neuronal activity. Neuroscience 70:893-924.

Raz A, Vaadia E, Bergman H (2000) Firing patterns and correlations of spontaneous discharge of pallidal neurons in the normal and the tremulous 1-methyl-4-phenyl-1,2,3,6-tetrahydropyridine vervet model of parkinsonism. J Neurosci 20:8559-8571.

Rosenberg JR, Amjad AM, Breeze P, Brillinger DR, Halliday DM (1989) The Fourier approach to the identification of functional coupling between neuronal spike trains. Prog Biophys Mol Biol 53:1-31.

Schultz W (1982) Depletion of dopamine in the striatum as an experimental model of parkinsonism: direct effects and adaptive mechanisms. Prog Neurobiol 18:121-166.

Smith Y, Bevan MD, Shink E, Bolam JP (1998) Microcircuitry of the direct and indirect pathways of the basal ganglia. Neuroscience 86:353-387.

Steriade M (1999) Coherent oscillations and short-term plasticity in corticothalamic networks. Trends Neurosci 22:337-345.

Stern EA, Kincaid AE, Wilson CJ (1997) Spontaneous subthreshold membrane potential fluctuations and action potential variability of rat corticostriatal and striatal neurons in vivo. J Neurophysiol 77:1697-1715.

Tseng KY, Riquelme LA, Belforte JE, Pazo JH, Murer MG (2000) Substantia nigra pars reticulata units from 6-hydroxydopamine-lesioned rats differ in their response to striatal D2 dopamine receptor stimulation and subthalamic lesions. Eur J Neurosci 12:247-256.

Tseng KY, Kasanetz F, Kargieman L, Pazo JH, Murer MG, Riquelme LA (2001) Subthalamic nucleus lesions reduce low frequency oscillatory firing of substantia nigra pars reticulata neurons in a rat model of Parkinson's disease. Brain Res, in press.

Umemiya M, Raymond LA (1997) Dopaminergic modulation of excitatory postsynaptic currents in rat neostriatal neurons. J Neurophysiol $78: 1254-1255$.

Urbain N, Gervasoni D, Souliere F, Lobo L, Rentero N, Windels F, Astier B, Savasta M, Fort P, Renaud B, Luppi PH, Chouvet G (2000) Unrelated course of subthalamic nucleus and globus pallidus neuronal activities across vigilance states in the rat. Eur J Neurosci 12:3361-3374.

Waszczak BL, Martin LP, Grief GJ, Freedman JE (1998) Expression of a dopamine $\mathrm{D} 2$ receptor-activated $\mathrm{K}+$ channel on identified striatopallidal and striatonigral neurons. Proc Natl Acad Sci USA 95:11440-11444.

Wichmann T, Bergman H, Starr PA, Subramanian T, Watts RL, DeLong MR (1999) Comparison of MPTP-induced changes in spontaneous neuronal discharge in the internal pallidal segment and in the substantia nigra pars reticulata in primates. Exp Brain Res 125:397-409.

Wickens JR, Wilson CJ (1998) Regulation of action-potential firing in spiny neurons of the rat neostriatum in vivo. J Neurophysiol 79:2358-2364.

Wilson CJ (1993) The generation of natural firing patterns in neostriatal neurons. Prog Brain Res 99:277-297.

Wilson CJ, Groves PM (1981) Spontaneous firing patterns of identified spiny neurons in the rat neostriatum. Brain Res 220:67-80.

Wilson CJ, Chang HT, Kitai ST (1983) Disfacilitation and long-lasting inhibition of neostriatal neurons in the rat. Exp Brain Res 51:227-235. 